\title{
PEX12 Novel Mutation: A Case Report on Iranian Girl with Childhood Onset Peroxisomal Disorder: Pseudo ALD?
}

\author{
Karimzadeh $\mathrm{P}^{1}$, Najmabadi $\mathrm{H}^{2}$, Tabarestani $\mathrm{S}^{1 *}$, Ahmadabadi $\mathrm{F}^{3}$, Ahangari $\mathrm{F}^{4}$, Fadaee $\mathrm{M}^{4}$, and Farokhashtiani $\mathrm{T}^{1}$ \\ ${ }^{1}$ Department of pediatrics, Shahid Beheshti University of Medical Sciences (SBMU), Iran \\ ${ }^{2}$ Genetics Research Center, University of Social Welfare and Rehabilitation Science, Iran \\ ${ }^{3}$ Departments of Pediatrics, Ardabil University of Medical Sciences, Iran \\ ${ }^{4}$ Kariminejad-Najmabadi Pathology \& Genetics Center, Iran \\ *Corresponding author: Sepideh Tabarestani, Pediatric Neurologist, Pediatric Neurology Research center, Shahid Beheshti University of Medical \\ Sciences (SBMU), Mofid Children Hospital, Tehran, Iran
}

Submission: 眥 October 29, 2017; Published: 眥 March 23, 2018

\begin{abstract}
Objective: To report a 7 years old girl with genetic and biochemical characteristics of zellweger spectrum disorder who presented with phenotype and neuro imaging findings of adrenoleukodystrophy.

Methods: Target gene sequencing was performed by using a custom designed panel for this patient with focused mutation analysis on 16 peroxisomal genes included in the panel. Sanger sequencing was used to confirm the identified mutation.

Results: This patient was homozygote for a missense mutation in exon 2 of the PEX 12 gene; c.541T>G (p.Tyr181Asp) which is a conserved residue in the N-terminal region crucial for localization to peroxisomes. Her parents and healthy sister were also found to have the same heterozygote mutation.

Conclusion: We suggest that PEX 12 gene mutations with a milder phenotype may be a potential candidate for posterior dominant leukodystrophy of childhood resembling X-linked ALD. This is entitled to pseudo- ALD and may be a diagnostic possibility in patients who develop ALD phenotype and neuroimaging particularly, in girls.
\end{abstract}

Keywords: Zellweger spectrum disorder; Childhood onset; Pseudo-ALD; PEX 12 gene; Novel mutation

Abbreviations: PBD: Peroxisomal Biogenesis Disorders; ZS: Zellweger Syndrome; NALD: Neonatal Adrenoleukodystrophy; IRD: Infantile Refsum Disease

\section{Introduction}

Peroxisomal Biogenesis Disorders (PBDs) are peroxisomal disorders presenting as a clinical continuum consists of at least three phenotypes; Zellweger Syndrome (ZS), Neonatal adrenoleukodystrophy (NALD) and Infantile Refsum Disease (IRD) with ZS being the most and IRD the least severe presentation. The PEX genes, which encode proteins called peroxins, are involved in ZSDs pathogenesis. Type of mutation and dysfunction of affected peroxins will determine disease severity and clinical phenotype. Mutations in PEX1 are the most common cause of ZSD and account for $70 \%$ of the cases. PEX6 and PEX12 mutations comprise $16 \%$ and $9 \%$, as the second and third common causes of ZSDs, respectively [1].

We present a female patient with a new mutation in PEX12 gene, and her clinical, biochemical assay and neuro imaging will be discussed. Ethical approval for this study was obtained by Pediatric neurology Research Center of Shahid Beheshti University of Medical sciences (SBMU).

\section{Case Report}

A seven-year old girl was referred to Pediatric Neurology Department of Mofid Children Hospital, Tehran, Iran, for regression in all neuro developmental milestones. She was delivered by an uneventful cesarean section at full term from consanguineous healthy parents of Turkish decent. There was no history of neonatal jaundice, however; she was admitted for 2 days because of restlessness. There was no history of feeding difficulty and her growth rate was within the normal range. She began walking at 17 months old and talking on time and appropriately (parental report). Her parents didn't notice any issues up to her fifth birthday when she started having slowly progressive appendicular and truncal ataxia. Dysarthria presented shortly after the ataxia, and her cognition gradually declined. 
At the age of 6 , she was unable to walk with speech ability limited to 1-2 words. Swallowing difficulties was followed at the age of 6.5. Her parents were first cousins. Her family history was positive for two cases of mental retardation and seizure disorder in addition to one death due to an unknown neurodegenerative disease. On examination, there were no dysmorphic features or organomegaly. Her neurological examination revealed spasticity in upper and lower extremities, and exaggerated deep tendon reflexes in the bedridden girl. The Ophthalmologic report was normal without any cataract or retinopathy, although she didn't have proper fix and follow. Auditory Brain Stem response confirmed severe bilateral sensor neural hearing loss. All Liver enzymes and function, electrolytes, kidney, adrenal and thyroid tests returned normal. Abdominal ultrasound, echocardiography, nerve conduction velocity and electromyography were also reported in normal range.
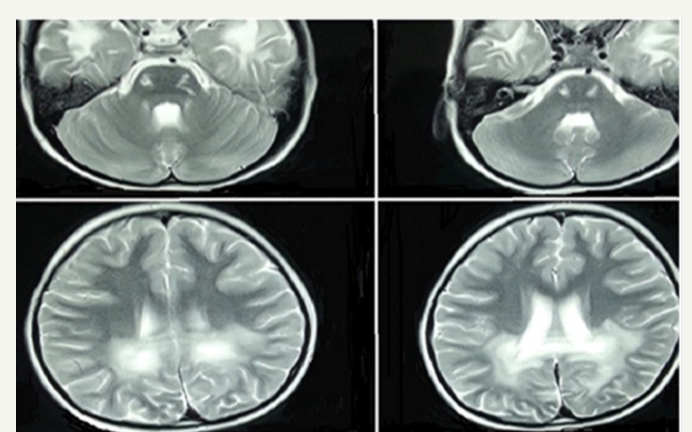

Figure 1: MR T2 images show corticospianl tracts involvement that spare cerebellum (Upper row), hyper intensities in posterior white matter with different zones characteristic for ALD. (Lower row)

MRI at the age of 5 revealed severe signal changes in per ventricular white matter of bilateral posterior occipital regions. There was no neocortical dysplasia or atrophy, and the cerebellum was normal. However, splenuim of corpus collosom and corticospinal tracts were affected (Figure 1). Different zones (ALDlike) were clearly differentiated on T2 MR Image. Second MRI at the age of 7 revealed extensive white matter involvement along with generalized atrophy. MRS demonstrated a choline rise in respect to NAA (N-Acetyl Aspartic Acid) compatible with leukodystrophy pattern.

Table 1: Summary of biochemical assay of the patient.

\begin{tabular}{|c|c|c|}
\hline Metabolite (Plasma) & Result & Reference \\
\hline $\begin{array}{c}\text { Hexacosanoic acid } \\
\text { (C-26) }\end{array}$ & $4.5 \mathrm{~nm} / \mathrm{ml}$ & $0.2-1.6$ \\
\hline $\begin{array}{c}\text { Tetracosahexaenoic } \\
\text { acid(C-24) }\end{array}$ & $122.4 \mathrm{~nm} / \mathrm{ml}$ & $12.0-94.0$ \\
\hline $\begin{array}{c}\text { Docosanoic acid } \\
\text { (C-22) }\end{array}$ & $41.8 \mathrm{~nm} / \mathrm{ml}$ & $15.0-113.0$ \\
\hline C24/C22 & 2.99 & $0.55-1.05$ \\
\hline C26/C22 & 0.109 & $0.005-0.029$ \\
\hline Phytanic acid & $235.8 \mathrm{~nm} / \mathrm{ml}$ & $0.3-31.0$ \\
\hline Pristanic acid & $118.9 \mathrm{~nm} / \mathrm{ml}$ & $0.0-3.0$ \\
\hline
\end{tabular}

Plasma ammonia, lactate and pyruvate were within normal range. Biochemical assay ruled out MLD and Krabbe with normal galactocerebrosidase and arylsulfatase A assay. ß-glucocerebrosidase assay, urine organic acid and plasma amino acid (HPLC) were normal, and celiac tests were negative. VLCFA assay showed a pattern observed in ZDSs; elevation of C26, Phytanic Acid and Pristatic acid in plasma, and increased C24/C22 and C26/ C22 ratios. These tests were sent to the Wagner stibbe referral laboratory in Gottingen/Germany and repeated twice (Table 1).

\section{Genetic Study}

The Germline DNA of the patient, her healthy sister and parents were extracted from white blood cells by salting out method. Target gene sequencing was performed by using a custom designed panel for this patient with focused mutation analysis on 16 peroxisomal genes included in the panel. In order to remove the common and benign variants, filtering steps were performed on the detected variants (MAF> $1 \%$ in public database). Benign synonymous and missense variants were predicted by the bioinformatics prediction software (Mutation taster, PolyPhen2 and SIFT). All identified mutations were confirmed by Sanger sequencing.

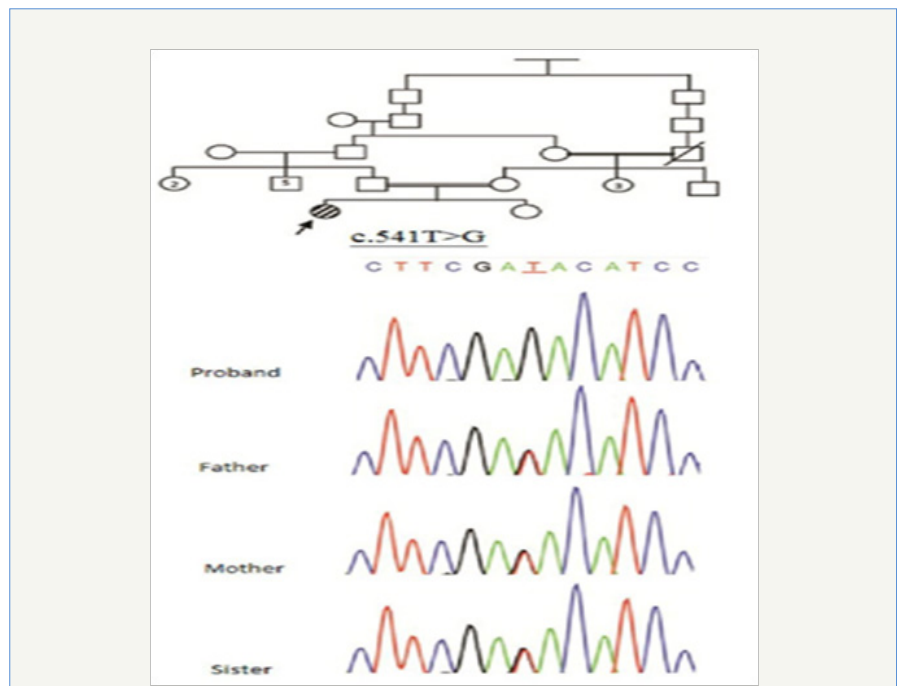

Figure 2: Pedigree and chromatograms which shows the mentioned mutation

The genetic testing confirmed that our patient is homozygote for a mutation defined as c.541T>G (p.Tyr181Asp) in exon 2 of PEX12 gene (NM-000286.2) which affects a conserved residue in the N-terminal region crucial for localization to peroxisomes. Clearly, the tyrosine residue in this position belongs to a polar class while aspartate is a charged (negative) amino acid. According to the software predictions, this mutation would not be tolerated in protein structure. Co-segregation of this variant has confirmed that our patients' parents and healthy sister are heterozygous for this variant (Figure 2).

To our knowledge, this variant has not been reported before; however, Steinberg et al. had reported a frame shift mutation at the same position of the protein (Tyr181Leufs*37) in two different studies in 2004 and 2009 causing Peroxisome biogenesis disorders [1]. 


\section{Discussion}

PEX12 gene mutations may present with milder phenotypes. However, we presented a novel mutation in PEX 12 gene with progressive leukodystrophy starting at the age of 5- year without any other organ involvement usually expected from ZSDs. She survived up to 8 years of age. The frame shift mutation of PEX12 in the same position as hers (541_542 insT) is reported as heterozygote allele of ZS phenotype in at least two studies [1] but her missense mutation demonstrates X-ALD phenotype which is a single enzyme/protein deficiency disorder.

Historically, two other single peroxisomal enzyme deficiency including peroxisomal acyl-CoA oxidase 1 deficiency (in 1998) and D-bifunctional protein deficiency (in 1987) were first described as pseudo-neonatal adrenoleukodystrophy and pseudo-ZD (recently named ZD-like disease) respectively, because of the similar sign and symptoms yet different pathogenesis [2]. Besides, it was previously noticed that male patients with late onset ZSD may be theoretically misdiagnosed as X-ALD. The reported patients however, had mostly PEX1 or PEX6 mutations or no genetic study had been done for them [3]. We didn't find any report of PEX 12 mutations to have both ALD phenotype and Neuro imaging that might show the rarity or under diagnosed cases. PEX gene mutations may occur in some particular ethnic backgrounds and therefore not attract enough attention. Moreover, complex PEX12 interac tions with other PEX proteins may affect the diagnostic procedure. For instances, one study showed that over expression of either PEX5 or PEX 10 can suppress S320F mutation in PEX 12 [4].

Previous reported phenotypes associated with PEX12 mutations include ZS, NALD, IRD presenting in different age range and severity, SMA-like disorder [4], and ZD with severe mitochondrial myopathy [5]. These similarities and "pseudo concepts" may suggest a common process that can be translated to a general therapeutic strategy.
In our report however, we were not able to perform ERG, ruling out possible asymptomatic retinal involvement, neither VEP nor skeletal survey given our patient clinical condition and nondiagnostic nature of these assessments. We didn't also perform the functional study; it may uncover some new aspects of PEX12 gene function.

\section{Conclusion}

We suggest that PEX12 gene mutations could be a potential candidate in posterior dominant leukodystrophy resembling $\mathrm{X}$-ALD. This is entitled to pseudo- ALD and may be a diagnostic possibility in patients who develop ALD phenotype and Neuro imaging particularly, in girls. Further studies are required for better identification of PEX12 mutations, genotype- phenotype coloration and its significance in leukodystrophy of childhood.

\section{References}

1. Yik WY, Steinberg SJ, Moser AB, Moser HW, Hacia JC (2009) Identification of Novel Mutations and Sequence Variation in the Zellweger Syndrome Spectrum of Peroxisome Biogenesis Disorders. Hum Mutat 30(3): 467480.

2. Poll-The BT, Gärtner J (2012) Clinical diagnosis, biochemical findings and MRI spectrum of peroxisomal disorders. Biochim Biophys Acta 1822(9): 1421-1429.

3. Weller S, Rosewich H, Gartner J (2008) Cerebral MRI as a valuable diagnostic tool in Zellweger spectrum patients. J Inherit Metab Dis 31(2): 270-280.

4. Chang CC, Warren DS, Sacksteder KA, Gould SJ (1999) PEX12 Interacts with PEX5 and PEX10 and Acts Downstream of Receptor Docking in Peroxisomal Matrix Protein Import. The Journal of Cell Biology 147(4): 761-774.

5. Salpietro V, Phadke R, Saggar A, Hargreaves LP, Yates R, et al (2015) Zellweger syndrome and secondary mitochondrial myopathy. Eur J Pediatr 174(4): 557-563.
Creative Commons Attribution 4.0 International License

For possible submissions Click Here
Submit Article

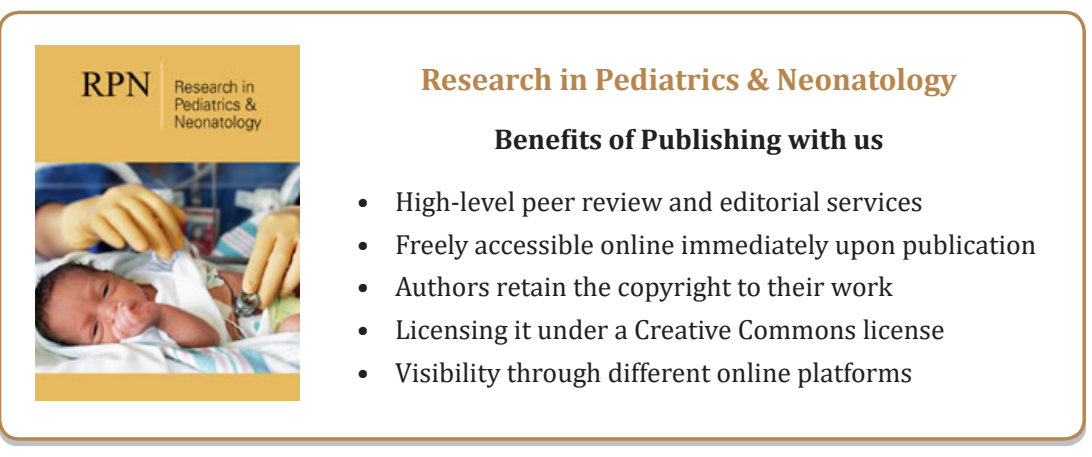

